\title{
DISEÑO METODOLÓGICO PARA LA ELABORACIÓN DE UNA ESTADÍSTICA SOBRE INNOVACIÓN TECNOLÓGICA DE DIMENSIÓN REGIONAL. SU APLICACIÓN EN ANDALUCÍA
}

\author{
Rosa Jordá Borrel, J. Carlos Posada Simeón y Ángel Luis Lucendo Monedero \\ Departamento de Geografía. Universidad de Sevilla, María de Padilla s/n, Sevilla, \\ e-mail: borrell@cica.es
}

\begin{abstract}
Resumen: El estudio de la innovación en la empresa y en el espacio se ha convertido en un tema de investigación fundamental para el conocimiento de la influencia del cambio tecnológico en el desarrollo de las regiones y en la llamada Geografía de la Organización. Precisamente la existencia en el ámbito regional de importantes déficits en materia estadística, dificultades metodológicas, escasez de estudios específicos o, incluso, el desconocimiento del número real de empresas innovadoras, nos lleva a exponer, a partir de este trabajo, la necesidad de aportar una metodología para la elaboración de una estadística sobre innovación para cualquier Comunidad Autónoma, aunque en este caso se aplique a Andalucía. De esta forma pretendemos diseñar unas directrices metodológicas para conocer la estructura y el alcance de los impactos de los procesos innovadores seguidos por las empresas andaluzas. Al mismo tiempo, plantear la construcción de estadísticas en dicha materia que sean comparables en el ámbito autonómico, nacional e internacional. Y por último, esbozar diferentes escenarios o propuestas metodológicas, según los casos, para la realización de esta estadística.
\end{abstract}

Palabras Clave: Metodología, estadística regional, innovación, Andalucía

\begin{abstract}
Studying innovation in a company has become a topic of fundamental research in order to understand the influence of technological change in the development of regions. Indeed, the fact there are important deficits in statistical matters, methodological difficulties, scarcity of specific studies or even, a lack of knowledge of the actual amount of innovative companies at a regional level, leads us to reveal in our paper the need to provide a methodology to draft statistics on innovation in Andalusia. This way, we intend to develop methodological guidelines that will allow us to understand the structure and effects of innovative processes implemented by Andalusian companies. At the same time, our intention is to set forth the development of statistics in this area, which could be comparable at a regional, national and international level. Our final aim is to create different scenarios or methodological proposals depending on the cases to develop these statistics.
\end{abstract}

Keywords: methodology, regional statistics, innovation, Andahusia. 


\section{INTRODUCCIÓN}

A lo largo de los últimos veinte años, la innovación y el cambio tecnológico se han convertido en temas cada vez más importantes en el análisis económico y en la toma de decisiones políticas, de manera que su interrelación con la riqueza y el crecimiento económico han situado a la dimerisión regional de estos parámetros en el centro de atención de los investigadores. Así, el estudio de la innovación en la empresa y en el espacio se ha constituido en una materia de investigación fundamental para el conocimiento del impacto del proceso de innovación en el desarrollo de las regiones y en la Geografía de la Organización. Sin embargo, la ausencia de datos sistemáticos fiables sobre esta cuestión, la escasez de análisis específicos o, incluso, el desconocimiento del número real de empresas innovadoras dificultan el estudio de la innovación empresarial de los ámbitos locales que no sólo tiene influencia en el desarrollo regional, sino que repercute en la economía de todo el país.

Estas carencias nos llevan a plantear, como objetivo principal del artículo, la realización de un diseño metodológico de la estadística de innovación tecnológica de las empresas andaluzas con un enfoque sistémico; que, además, nos proporcione un conocimiento general sobre la estructura y los efectos de los procesos innovadores de las firmas en el ámbito territorial. Al mismo tiempo, diseñar estadísticas que midan la importancia del espacio relacional construido y a la vez que sean comparables a distintas escalas (ámbito autonómico, nacional e internacional), tal y como proponen las directrices de la OCDE y EUROSTAT. Y por último, esbozar diferentes escenarios o propuestas metodológicas, según los casos, para llevar a cabo estas estadísticas.

\section{METODOLOGÍAS DE LA ESTADÍSTICA SOBRE INNOVACIÓN}

La elaboración de unas estadísticas sobre innovación de dimensión regional y espacial requiere desarrollar un proceso metodológico y un cuerpo teórico inexistentes hasta el momento. En España, los Institutos Estadísticos de las CCAA adolecen de tradición y de recursos económicos y humanos para desarrollar unas estadísticas sobre innovación e $\mathrm{I}+\mathrm{D}$ basadas en información exclusivamente regional que midan los impactos territoriales, como después veremos. Hasta ahora, las ciencias sociales, y en especial la Geografía, han avanzado poco en el terreno estadístico de la innovación y en el caso de estadísticas oficiales de ámbito regional los estudios son inexistentes. Por otra parte, esta estadística debe estar normalizada y homologada para establecer la comparación a todas las escalas. Los organismos que marcan las pautas para la realización de estadísticas sobre innovación son la OCDE y EUROSTAT, si bien conviene llamar la atención que éstos han dictado directrices fundamentalmente para poner en marcha estadísticas de carácter estatal, salvo en el caso de la publicación del Manual Regional. 
Las instituciones estadísticas de los países occidentales siguen estas pautas adoptando como norma los manuales de Frascati y de Oslo. En España, el organismo estatal que asume esas metodologías internacionales es el INE. Por lo tanto, el camino a seguir para la elaboración de unas estadísticas sobre innovación regional sería ver las orientaciones esbozadas a escala internacional; luego, analizar la metodología seguida por el INE; y después descender al nivel regional para comprobar si existen algunas en este ámbito territorial. Finalmente, conocer las líneas que marcan todos estos organismos para poder ser aplicadas en Andalucía.

A escala internacional, los primeros antecedentes estadísticos sobre los estudios de innovación se sitúan en los años 60 , centrándose únicamente en las estadísticas de I+D. Pero, a partir de las sucesivas ampliaciones y modificaciones del Manual Frascati (OCDE, 1963 y 1993) ${ }^{1}$ se llegó a la conclusión de que la innovación es un proceso complejo que sobrepasa los límites marcados por las actividades de $\mathrm{I}+\mathrm{D}$, especialmente por aquéllas que tienen que ver con los descubrimientos científicos. Pues estas estadísticas sólo serían aplicable a aquellas regiones que tienen una base científica y tecnológica muy avanzada y que son capaces de desarrollar tecnologías complejas regionalizadas (STORPER, 1995) desembocando éstas en cambios radicales globales.

Tales hechos originaron la redacción de un nuevo manual que planteara un cambio metodológico y un estudio del proceso innovador apoyado en la demanda del mercado, en el análisis del output y en el estudio de redes, dando lugar al Manual de Oslo y al Manual Regional (OCDE, 1992). De esta forma el método lineal seguido por el Manual de Frascati fue superado por el denominado de enlace en cadena -o modelo interactivo- (KLINE Y ROSENBERG, 1986), el cual englobaría la dimensión sistémica de la innovación realizada por las grandes empresas, las PYMEs, los centros de investigación, organismos tecnológicos, etc. lo que daría lugar a un modelo aplicable a cualquier tipo de región, independientemente del nivel innovador en que se encuentre.

Por su parte, la Oficina Estadística de las Comunidades Europeas (EUROSTAT) inició diversos estudios encaminados a la recogida de datos sobre innovación en sus Estados miembros, en coordinación con la OCDE, y para ello presentó una propuesta de decisión del Consejo sobre estadísticas de $\mathrm{I}+\mathrm{D}$ e innovación, que fue aprobada en enero de 1994. En ese mismo año, para responder a estas necesidades, el INE empezó a elaborar la Encuesta sobre Innovación Tecnológica en la Empresa (INE, 1998 y 2000).

Dicho estudio ha permitido la obtención de unos indicadores que nos aproximan al conocimiento de la estructura y de los efectos del proceso innovador desde el punto de vista sectorial y espacial a escala nacional, así como de disponer de un marco para futuras investigaciones. Con la publicación de los resultados del INE de 1996 se da continuidad a estos trabajos que posteriormente se han visto ampliados en los de 1998 y 2000 . Éstas últimas incorporan nueva información sobre la innova- 
ción tecnológica en España, enriqueciendo la dimensión espacial de esa estadística a través de la publicación de indicadores que miden sus impactos, sin embargo salvo en una ocasión no aparece información regionalizada. Por consiguiente, para la elaboración de una metodología sobre estadísticas de actividades innovadoras de ámbito regional es necesario analizar las orientaciones teóricas existentes en las tres escalas antes reseñadas (internacional, nacional y regional), lo que realizaremos en los apartados siguientes.

\section{DIRECTRICES INTERNACIONALES SOBRE LA ESTADÍSTICA DE INNOVACIÓN APLICADA A REGIONES}

Tal como hemos visto antes, el proceso de innovación del modelo lineal evolucionaba pasando por distintas etapas: investigación, invención, innovación y difusión. A su vez, la investigación se desarrollaba a través de diversas fases: conocimientos científicos básicos, tecnológicos e ingeniería práctica. Por ello, debido a esta hipótesis de linealidad, la $\mathrm{I}+\mathrm{D}$ se consideraba el principal indicador de las actividades de innovación, siendo difícil introducir la dimensión espacial.

Así, las estadísticas disponibles para el análisis de la innovación se podían resumir en tres grandes categorías: inputs en $\mathrm{I}+\mathrm{D}$, patentes e indicadores bibliométricos. Las limitaciones de estos datos son bien conocidas. Por un lado, las cifras de I+D no tienen en cuenta los resultados de la innovación y, por otro, tienden a subestimar la actividad innovadora de las pequeñas empresas, ya que numerosas firmas innovan dedicando pocos recursos a I+D. La información sobre patentes es insuficiente porque no todas las firmas siguen la misma política en materia de depósitos. Además, éstas se tefieren a la fase de invención pero casi nunca aluden a la de comercialización y, por tanto, no aparece reflejado el impacto económico de la misma. Por otra parte, tienden a menospreciar el proceso innovador de las grandes empresas ya que éste es mucho más amplio que la actividad de I+D patentada. Por último, los datos bibliométricos se centran en la investigación básica, pero no en el proceso de innovación.

Por ello, el marco teórico que inspira el Manual de Oslo, el de Kline y Rosenberg, reconoce no sólo la organización sistémica del proceso innovador, sino también las principales especificidades del modelo lineal y además incorpora las aportaciones más relevantes de la teoría del cambio tecnológico procedentes del pensamiento evolucionista (Nelson y Winter, 1982, Dosi,1992), tales como la existencia de mecanismos de selección, el carácter acumulativo del conocimiento, la diversidad de trayectorias, la definición de la tecnología como compleja, diferenciada y multifuncional, etc. De ahí que este manual haga ver a sus lectores que la innovación tecnológica es el resultado de las características del entorno regional cuya estrategia se centra en la trans- 
formación de la estructura productiva, en el proceso de cambio de las empresas y, en definitiva, en la construcción de un nuevo espacio relacional (Santos, M., 2000).

De esta forma, en el diseño de la encuesta, recomendado por el Manual, se tiene en cuenta los vínculos de la firma con el resto de los elementos del sistema nacional de innovación incluyendo en las unidades productivas a proveedores y clientes, instituciones, centros de investigación, mercados, etc. poniendo de manifiesto la importancia de la transferencia y de la difusión de innovaciones (información, conocimiento, etc.) en el espacio (Morril, Gaile y Thrall, 1988). Igualmente, se hace hincapié en las vías y las redes a través de las cuales circulan la información y el conocimiento, factores éstos muy importantes para medir el aprendizaje tecnológico y el alcance de los spillovers o fenómeno del desparramamiento, resultantes del desarrollo del sistema ciencia tecnología industria nacional/ regional. Es decir, el Manual de Oslo adopta, por una parte, las ideas de Lundvall (1992) el cual considera que un sistema de innovación está constituido por elementos y relaciones interactuantes en el marco de la producción, de la difusión y de la utilización de nuevos conocimientos y económicamente útiles. Por otra, retoma el enfoque del modelo de Porter y de la geografía de la organización (Veltz, 2000) al considerar el territorio como una matriz de organización e interacciones sociales viendo lo que sucede entre los actores tecnológicos del espacio (entre empresas y entre empresas e instituciones).

En consecuencia, cuando se sigue las directrices del Manual de Oslo cabe medir: a) los inputs del proceso innovador o análisis de gastos; b) los impactos que tienen lugar en las empresas y en el espacio resultante derivado de las inversiones en nuevos y mejores productos y procesos; c) el análisis de la difusión territorial de las innovaciones en el mismo sector y en otras actividades empresariales; d) y por úitimo, es posible abordar el efecto producido por las innovaciones no tecnológicas como la cooperación, las de organización y las de gestión de calidad, desde el punto de vista sectorial y espacial. Poniendo de manifiesto que las innovaciones conllevan una serie de actividades sistémicas científicas, tecnológicas, organizativas, financieras y comerciales. Todo lo cual desemboca en la construcción de un espacio social complejo que nos puede llevar incluso a la formación de polos donde domine la llamada economía del conocimiento.

Pero si la aportación del Manual de Oslo consiste en incorporar a la innovación tecnológica las teorías evolucionistas, de redes, de la geografía de la organización y sistémicas, y en mostrar como se deben recoger los datos de ámbito estatal, el Manual Regional nos orienta sobre las posibilidades de hacer unas estadísticas específicas de dimensión regional comparables a otras escalas, sobre las bases teóricas que acabamos de mencionar. Dicha homologación presupone la obligatoriedad de usar conceptos comunes y equiparables. Sin embargo, el problema más importante que se plantea a este nivel es que la recogida de datos está menos perfeccionada que a escala nacional donde existe una mayor tradición. En este contexto, el Manual Regional nos llama la atención de que no basta con utilizar conceptos e indicadores 
establecidos en el ámbito nacional, sino que debe tenerse en cuenta las particularidades de la dimensión regional (Cano García, 1987). Así entre las principales directrices que nos sugieren destacan las siguientes:

- La unidad informante (entidad de la cual se recogen los elementos de información deseados) referente a la empresa a escala regional debe ser la unidad local (UL) o, en caso de necesidad de un análisis por actividad económica, la unidad de actividad económica al nivel local (UAE local). La UL es una empresa o parte de empresa sita en un lugar delimitado topográficamente.

- Los procedimientos para establecer indicadores regionales de innovación pueden seguir distintos métodos. El método ascendente (bottom up) basa la estimación de los indicadores regionales en la información disponible en el ámbito de la UAE local o UL que se agrega hasta que se establece el nivel regional considerado. Este método tiene la ventaja de que se apoya directamente en datos regionales y la agregación puede detenerse en cualquier nivel regional intermedio. Pero a pesar de esta cualidad el coste económico sería excesivo para una región como la nuestra como después veremos. El otro procedimiento planteado es el descendente, el cual se centra en la distribución de una cifra nacional entre las regiones, sin intentar distinguir la UL de la UAE local, por medio de una clave de distribución que refleja lo más fidedignamente posible la variable que ha de estimarse. La principal ventaja de esta forma de recoger la información es la coherencia numérica entre los indicadores nacionales y regionales (Camagni, 1991). En su contra tiene que las estimaciones no se basan directamente en datos regionales. Junto a estos métodos aparecen múltiples procedimientos mixtos como resultado de las combinaciones de los métodos ascendentes y descendentes puros en los diferentes niveles de agregación.

- El desglose por actividad económica es importante para las estadísticas de innovación en el sector empresarial (Felman, 1994). Normalmente se clasifican las empresas por su actividad principal. Esta propuesta es aconsejable en el ámbito nacional, planteando problemas a escala regional. Así, empresas que participan en más de una actividad económica suelen concentrar las actividades en regiones diferentes, no ejerciendo cada una de las UL todas las actividades económicas de la empresa. Este hecho puede asociar a las UL actividades que realmente no realizan.

- Por último, es conveniente tener en cuenta que la agregación a escala nacional de la información obtenida de la UL puede conducir a resultados diferentes de los recogidos al nivel de empresas.

En consecuencia, existen como mínimo dos posibles procedimientos para desarrollar unas estadísticas regionales de innovación. El método ascendente o estadísticas basadas en datos regionales supone poner en marcha una encuesta de innova- 
ción propia lo que implica elaborar un directorio de empresas, un estudio muestral, etc (Patel y Pavitt, 1995). Junto a estas dos actividades este proceso conlleva ciertas dificultades:

- resulta difícil comparar estas estadísticas con las de tipo nacional e internacional.

- el desarrollo de tareas de recogida de información es problemática por falta de tradición, duplicidades entre varios organismos, etc.

- debido a su complejidad, la elaboración de estas estadísticas puede tener un alto coste, exigiendo al organismo encargado tener un mínimo de recursos humanos especializados y dedicados casi exclusivamente a esa materia ${ }^{2}$; además de poseer una infraestructura técnica que posibilite las tareas de elaboración, recogida de información, tratamiento, explotación... de los datos.

Toda esta problemática derivada de la aplicación del método ascendente nos lleva a seguir como ejercicio de investigación un camino alternativo que se adapte mejor a la disponibilidad de medios financieros y humanos con los que cuenta la región objeto de estudio (Archibugi, 1997), en este caso Andalucía. Ni siquiera otras comunidades autónomas con un volumen de recursos mayor, como el País Vasco o Cataluña, se plantean la elaboración de una estadística de innovación propia siguiendo esta metodología. De ahí que hayamos asumido fundamentalmente un proceso metodológico inverso desarrollado a partir del método descendente; si bien conviene subrayar que éste también presenta una serie de inconvenientes, entre los que destaca la necesidad de seguir la metodología de ámbito nacional adaptada a las peculiaridades regionales.

\section{PROBLEMÁTICA DE LA ESTADISTICA NACIONAL PARA EL ESTUDIO DE LA INNOVACIÓN EN ANDALUCÍA}

A escala nacional sólo el INE realiza estadísticas sobre innovación. De éstas cabe extraer los únicos datos existentes sobre actividades innovadoras para Andalucía. Sin embargo, el hecho de obtener información de dimensión regional de unas estadísticas realizadas a escala estatal, con todo lo que esto conlleva (cuerpo teórico, metodología, etc.), origina una problemática añadida que desvirtúa la realidad de las actividades innovadoras desarrolladas en el tejido productivo andaluz. Debido a:

a) Una inadecuada representatividad de la muestra nacional para Andalucía, que implica una selección incorrecta de empresas innovadoras andaluzas tanto por sectores económicos, como por tamaño empresarial. 
- La encuesta sobre innovación tecnológica del INE de 1998 se extiende a todas las empresas industriales españolas cuya principal actividad económica corresponde a las secciones $\mathrm{C}$ (industrias extractivas), D (industrias manufactureras) y E (producción y distribución de energía eléctrica, gas y agua) de la CNAE-93 así como las empresas de servicios de telecomunicación (CNAE 64.20). El INE parte de una población total de 10.000 empresas que clasifica en función de esos sectores y después selecciona, de forma sistemática y con arranque aleatorio, una muestra representativa de las empresas innovadoras españolas a las que encuestará, ordenándolas por tamaño y provincia.

- Esta selección supone que determinadas comunidades autónomas estén mejor representadas que otras debido al hecho de poseer un elevado número de empresas innovadoras de los anteriores sectores. Así, las firmas de Cataluña, el País Vasco o Madrid tendrán mayor probabilidad de ser elegidas por el INE que las andaluzas, donde ramas como Agricultura, ganadería, caza y selvicultura (A), Transporte (I), o Actividades inmobiliarias y de alquiler; servicios empresariales $(K)$ tienen una mayor representatividad y sin embargo, no constituyen ramas a seleccionar por el Instituto Nacional de Estadística (Tabla 1).

- Respecto al tamaño empresarial, el INE de una población inuestral de 10000 empresas industriales, escoge a todas las firmas de 200 o más empleados, mientras que para el ervamos la Tabla 2 , donde se compara los porcentajes de la muestra nacional y andaluza por tamaños, comprobamos que los referidos a los primeros intervalos (de 1 a 4 y de 4 a 19) tienen unos valores muy bajos en el caso de España, mientras la realidad andaluza es totalmresto de los estratos ( 1 a $4 ; 5$ a 19; 20 a 49 y 50 a 199) se prefija un error del $10 \%$ para la variable gastos de innovación. De nuevo, este hecho implica la selección de un menor número de empresas andaluzas ya que la estructura empresarial de nuestra comunidad autónoma difiere de la nacional. Si obsente contraria a estas cifras, ya que las estructura empresarial de nuestra región se caracteriza por el predominio de la micro y la pequeña empresa.

- También podemos ver que la estructura empresarial andaluza por tamaño según el número de empleados de los estratos superiores difiere de la nacional. Las empresas con 200 o más empleados son más numerosas en CC.AA. más desarrolladas (Cataluña, el País Vasco o Madrid) que la nuestra. Teniendo en cuenta que el INE escoge la totalidad de estas firmas, Andalucía queda menos caracterizada que otras regiones.

b) Junto a estas dos consecuencias que se derivan de la representatividad de la muestra del INE, la unidad informante tampoco es la adecuada. 
Tabla 1. Gastos en innovación y su distribución por actividades innovadoras según rama de actividad principal de la empresa. Año $1998^{3}$

\begin{tabular}{|c|c|c|c|c|c|}
\hline \multirow[b]{2}{*}{ Rama de actividad } & \multicolumn{2}{|c|}{ ESPAÑA } & \multicolumn{2}{|c|}{ ANDALUCIA } & \multirow[b]{2}{*}{$\begin{array}{c}\text { ANDALUCÍA } \\
\text { ESPAÑA } \\
\%\end{array}$} \\
\hline & $\begin{array}{l}\text { Gastos en } \\
\text { innovación } \\
\text { (mill. de Ptas.) }\end{array}$ & $\%$ & $\begin{array}{c}\text { Gastos en } \\
\text { innovación } \\
\text { (INE) } \\
\text { (mill. de Ptas.) }\end{array}$ & 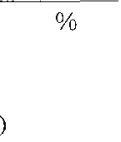 & \\
\hline Aeroespacial & 28.507 & $3,6 \%$ & 4.246 & $10,8 \%$ & 14,9 \\
\hline Alimentación, bebidas & 77.142 & $9,7 \%$ & 7.566 & $19,3 \%$ & 9,8 \\
\hline Aparatos de radio, $\mathrm{t}$, y comunicación & 47.822 & $6,0 \%$ & 1.199 & $3,1 \%$ & 2,5 \\
\hline Automóviles & 128.405 & $16,1 \%$ & 808 & $2,1 \%$ & 0,6 \\
\hline Cartón y papel & 19.852 & $2,5 \%$ & 575 & $1,5 \%$ & 2,9 \\
\hline Caucho y plástico & 24.728 & $3,1 \%$ & 3.670 & $9,3 \%$ & 14,8 \\
\hline Componentes electrónicos & 8.394 & $1,1 \%$ & 355 & $0,9 \%$ & 4,2 \\
\hline Coque, petróleo y combust. nuclear & 28.258 & $3,6 \%$ & 43 & $0,1 \%$ & 0,2 \\
\hline Cuero y calzado & 3.818 & $0,5 \%$ & 92 & $0,2 \%$ & 2,4 \\
\hline Edición, impresión y reproducción & 22.718 & $2,9 \%$ & 1.220 & $3,1 \%$ & 5,4 \\
\hline Electricidad, gas y agua & 27.701 & $3,5 \%$ & 1.117 & $2,8 \%$ & 4,0 \\
\hline Extractivas & 3.883 & $0,5 \%$ & 1.307 & $3,3 \%$ & 33,7 \\
\hline Farmacia & 54.739 & $6,9 \%$ & 919 & $2,3 \%$ & 1,7 \\
\hline Instrumentos, óptica y relojería & 10.610 & $1,3 \%$ & 83 & $0,2 \%$ & 0,8 \\
\hline Madera y corcho (excepto muebles) & 10.320 & $1,3 \%$ & 407 & $1,0 \%$ & 3,9 \\
\hline Manufacturas metálicas & 32.211 & $4,1 \%$ & 632 & $1,6 \%$ & 2,0 \\
\hline Máq. de oficina, cálculo y ordenadores & 7.185 & $0,9 \%$ & 0 & $0,0 \%$ & 0,0 \\
\hline Maquinaria y equipo mecánico & 42.462 & $5,3 \%$ & 990 & $2,5 \%$ & 2,3 \\
\hline Máquinas eléctricas & 31.429 & $4,0 \%$ & 1.679 & $4,3 \%$ & 5,3 \\
\hline Metales férieos & 25.754 & $3,2 \%$ & 3.106 & $7,9 \%$ & 12,1 \\
\hline Metales no férreos & 4.791 & $0,6 \%$ & 397 & $1,0 \%$ & 8,3 \\
\hline Minerales no metálicos & 32.950 & $4,1 \%$ & 2.137 & $5,4 \%$ & 6,5 \\
\hline Muebles & 11.767 & $1,5 \%$ & 833 & $2,1 \%$ & 7,1 \\
\hline Naval & 12.348 & $1,6 \%$ & 1.951 & $5,0 \%$ & 15,8 \\
\hline Otras manufacturas & 5.675 & $0,7 \%$ & 139 & $0,4 \%$ & 2,5 \\
\hline Otro material de transporte & 8.499 & $1,1 \%$ & 1.213 & $3,1 \%$ & 14,3 \\
\hline Prendas de vestir y peletería & 7.346 & $0,9 \%$ & 590 & $1,5 \%$ & 8,0 \\
\hline Química (excepto farmacia) & 53.643 & $6,8 \%$ & 1.734 & $4,4 \%$ & 3,2 \\
\hline Reciclaje & 334 & $0,0 \%$ & 0 & $0,0 \%$ & 0,0 \\
\hline Tabaco & 7.436 & $0,9 \%$ & 192 & $0,5 \%$ & 2,6 \\
\hline Textiles & 13.471 & $1,7 \%$ & 83 & $0,2 \%$ & 0,6 \\
\hline TOTAL & 794.196 & $00,0 \%$ & 39.284 & $100,0 \%$ & 4,9 \\
\hline
\end{tabular}

Fuente: elaboración propia a partir del INE.

- El INE toma como unidad informante la empresa puesto que es la unidad más utilizada a nivel internacional y además ofrece mayor facilidad a efectos de recogida de información y menor coste económico. En Andalucía la situación es distinta, porque un porcentaje importante de la innovación que se lleva a cabo en nuestra región -el 37,8\% del total de gastos según la 
muestra de la encuesta del INE de 1998 (Jordá Borrell, Posada Simeón y Lucendo Monedero, 2000)- se genera en establecimientos de empresas con sede social fuera de Andalucía. Además, hay que tener en cuenta que en la Comunidad Autónoma Andaluza las empresas o los establecimientos de mayor tamaño (según número de empleados) son aquéllos que realizan más innovación. Por ello, la unidad informante más adecuada en Andalucía ha de ser los establecimientos innovadores, ya que así lo ratifica el porcentaje de establecimientos innovadores de mayor tamaño ( $>250$ empleados) suponiendo un $47,2 \%$ del total de empresas innovadoras en Andalucía.

A pesar de la problemática descrita en los párrafos anteriores, la Encuesta sobre Innovación Tecnológica del INE es la única estadística oficial para Andalucía de la que podemos extraer una serie de variables a fin construir una metodología con el objetivo de estudiar la innovación en Andalucía. Entre ellas destacan: El número de empresas innovadoras, la regionalización de los gastos de innovación e $I+D$, cifra de negocios y las exportaciones, Grado de novedad de las innovaciones, Utilización de nuevas tecnologias en la fabricación, Cooperación en $I+D$, Participación en programas de investigación y Otras actividades no tecnológicas. Por ello, cuando se disponen de los datos desagregados al nivel de empresa podemos abordar los impactos territoriales, las interrelaciones espaciales de cada una de estas variables ${ }^{4}$ y los flujos resultantes de carácter intrarregional e interregional. Eso sí, teniendo en cuenta que únicamente podremos analizar aquellas firmas que tengan su sede social en la CCAA objeto de estudio, con lo cual la muestra puede que no sea representativa de la población.

Además, para los casos de las empresas que realizan actividades no tecnológicas, se registra información complementaria sobre: Gastos en innovaciones no tecnológicas e impacto económico de la innovación no tecnológica, poniendo en relación esta última variable con la de los imputs en innovación y con la de los impactos que tienen lugar en las empresas y en el entorno productivo o espacio relacional.

Sin embargo, el INE a la hora de publicar los datos recogidos a través de la encuesta no regionaliza los resultados, salvo la información correspondiente a las ramas de actividad por lo que no es posible su explotación a escala regional. De ahí la necesidad de elaborar una estadística sobre innovación a nivel de Comunidad Autónoma.

\section{PROBLEMÁTICA DE LA ESTADISTICA REGIONAL PARA EL ESTUDIO DE LA INNOVACIÓN EN ANDALUCÍA}

La existencia de fuentes de información regional significa una vía complementaria para elaborar unas estadísticas sobre innovación en Andalucía, aprovechando al 
Tabla 2. Porcentaje de empresas innovadoras a nivel nacional y regional por tamaños según la muestra del INE

\begin{tabular}{lccccccc}
\hline & \multicolumn{7}{c}{ EMPLEADOS } \\
\hline & 1 a 4 & 4 a 19 & 20 a 49 & 50 a 199 & 200 o más & Total \\
\% Andalucía & 3,05 & 20,81 & 13,20 & 28,43 & 34,52 & 100 \\
\% España & 4,45 & 13,65 & 22,60 & 39,15 & 69,70 & 100 \\
\hline
\end{tabular}

Fuente: elaboración propia a partir del INE.

máximo la información que suministra el INE y siguiendo en parte el método descendente. Igualmente supone la mejor manera para elaborar un directorio de empresas de acuerdo con las características de la población empresarial y sectorial andaluza, sin apartarnos de las normas internacionales basadas en la consecución de la homologación y la normalización. Su desarrollo constituye el procedimiento mixto más viable frente a los métodos ascendente y descendente puros, presentando diversas alternativas en función del volumen de recursos disponibles; alternativas que expondremos en el próximo apartado.

De acuerdo con lo expuesto hasta ahora, convendría poner de relieve que:

a) la elaboración de un directorio de empresas innovadoras andaluzas sería el camino óptimo para conocer en profundidad las características de estas firmas (tamaños, sectores, tipo establecimiento, etc.) máxime cuando, además, presentan unos rasgos distintos a la población innovadora nacional; porque si se decidiese seleccionar la muestra de empresas a encuestar de forma aleatoria, prescindiendo de la creación de ese directorio tal como hace el INE a escala nacional, el número de encuestas a recoger de empresas innovadoras andaluzas sería muy pequeño, dada la escasez de firmas innovadoras existentes comparado con el total de empresas en Andalucía.

b) a la hora de elaborar ese directorio, las fuentes complementarias a la información que suministra el INE presentan dificultades, bien por su parcialidad (El Catálogo de Empresas de Servicios Avanzados en Andalucía -SERAVAN- sólo recoge empresas de servicios avanzados, CDTI se centra en la I+D, la Red Andalucía Innovación); o por contener errores en la información registrada (CDTI, El Inventario de Recursos Tecnológicos de Andalucía-IRTA-); o por no disponer de una información homogénea en sus respectivas estructuras. En cualquier caso, todas son incompletas, incluida el INE, ya que ninguna recoge la totalidad de empresas innovadoras andaluzas. Por consiguiente resultaría laboriosa la tarea de realizar y mantener un directorio de empresas de innovación para Andalucía. 
En consecuencia:

1. El desconocimiento real de la población total de empresas innovadoras andaluzas es uno de los principales inconvenientes para el estudio de la innovación en Andalucía. Además, es una referencia necesaria para valorar las muestras utilizadas en los estudios que realizan los distintos autores sobre la innovación en nuestra región.

2. Los estudios sobre innovación empresarial y espacial referidos a Andalucía son escasos: Castells \& Hall (1992), Jordá Borrell (1992), Blasco, González y Podadera (1998), Pomares (1998), Durán (1999) y Jordá Borrell (2000).

\section{PROPUESTAS METODOLÓGICAS PARA LA ELABORACIÓN DE UNA ESTADÍSTICA DE INNOVACIÓN EN LA C.A. DE ANDALUCÍA}

En base a todo lo expuesto y partiendo de la necesidad de elaborar un directorio que recoja las empresas innovadoras andaluzas, resulta conveniente señalar el camino metodológico a seguir para la elaboración de una estadística sobre innovación empresarial y espacial en Andalucía. A tal fin procederemos a llevar a cabo una serie de propuestas que se fundamentan en metodologías específicas. A partir de éstas se extraen variables y resultados estadísticos que definen el proceso de innovación de las empresas en Andalucía. Para ello, se realizará una valoración de sus ventajas e inconvenientes, atendiendo a su comparabilidad con la estadística nacional e internacional, su adecuación a la realidad innovadora andaluza o los recursos (técnicos, humanos ...) necesarios para su elaboración.

\subsection{Propuesta 1. Explotación de los resultados estadísticos sobre innovación tecnológica del INE}

Esta propuesta se basa en la explotación de los datos estadísticos sobre innovación tecnológica del INE para Andalucía que ha de plantearse en los siguientes términos:

VENTAJAS

- Temporalidad bianual de la estadística regional al igual que la del INE y coincidiendo en los mismos años. Esto permite la comparabilidad con la estadística nacional e internacional.

- Bajo coste de la estadística ya que los recursos económicos y humanos los asume el INE. 


\section{INCONVENIENTES}

- No contabilización del establecimiento como unidad de información, pues para el INE ésta es la empresa.

- Una necesaria coordinación con el INE para facilitar los datos correspondientes a Andalucía. Tal circunstancia plantea dos posibilidades: a) prescindir de los registros correspondientes a empresas con sede social fuera de Andalucía, pero con establecimientos en nuestra región, lo cual implicaría que los resultados presenten un sesgo muy importante ya que éstos tienen una gran significación económica e innovadora en Andalucía. $\mathrm{Y}$ b) incorporar todos los registros y explotar tan sólo aquellas variables susceptibles de ser regionalizadas. En cualquier caso esta última información aparece ya publicada en la estadística nacional. Suponiendo que el INE no facilitase los datos regionalizados y por establecimientos (ya que el INE dispone de información de variables que no publica), la estadística sobre la innovación empresarial y espacial en Andalucía quedaría mermada en un alto porcentaje.

- Sesgo en población muestral: número, tamaño y sectores de actividad de las empresas como ha quedado de manifiesto a lo largo de este artículo.

- Continuas modificaciones en la metodología puesto que el INE se va adaptando, cada vez más, a las directrices internacionales (concepto de innovación, incorporación de otras actividades económicas...).

- Desde una perspectiva espacial cabe diferenciar, por un lado, los datos publicados por el IEA (2001) a partir de los cuales no se puede hacer un análisis territorial y únicamente cabe darle un enfoque sectorial. De nuevo debemos recordar que la unidad informante utilizada por el IEA y por el INE es la empresa, de forma que cuando éstas últimas tienen distintas delegaciones/plantas en diferentes CCAA se toma como valor para cada región bien el porcentaje de los gastos de innovación regionalizados o bien se acumula el total de éstos en su sede central que es donde se recoge la información. Además conviene advertir que no siempre las firmas innovadoras realizan en todas sus plantas actividades de éstas características, lo que quiere decir que no se dispone de información a nivel de establecimiento. De ahí que estas sean las causas por las cuales el INE publica sólo una variable regionalizada.

- Por otro lado, si se dispone de los datos desagregados a nivel de empresa para una CCAA podemos abordar los impactos territoriales, las interrelaciones espaciales de cada una de las variables que propone el INE y los flujos resultantes de forma parcial. Eso sí, teniendo en cuenta que únicamente podremos analizar aquellas firmas que tengan su sede social en Andalucía, con lo cual la muestra, tal como hemos dicho antes, no sería representativa de la población ni 
desde el punto de vista del tamaño empresarial ni desde la perspectiva de la distribución sectorial y territorial.

\subsection{Propuesta 2. Mejora de los resultados estadísticos sobre innovación tecnológica del INE}

Esta propuesta plantea la mejora de los resultados estadísticos de la Encuesta de Innovación Tecnológica que realiza el INE, subsanando así en la medida de lo posible las deficiencias identificadas en la anterior metodología -propuesta 1-. La problemática subyacente de la explotación de la base de datos del INE sobre innovación tecnológica induce a la adopción de una serie de medidas, que parcialmente contribuirían a enriquecer la estadística para Andalucía del INE. Se trataría, por tanto, de proponer una solución intermedia que supondría la introducción de cambios puntuales en la metodología seguida por el INE en la elaboración de la estadística sobre innovación y repercutiría positivamente sobre los resultados regionales, con el consiguiente abaratamiento de costes de cara a generar una estadística propia.

\section{VENTAJAS}

- Adecuación de la población muestral a la realidad innovadora andaluza. Para ello es necesario aumentar el tamaño de la muestra utilizada por el INE para Andalucía desde tres aspectos: a) incremento del número total de empresas para esta Comunidad; b) incorporación de nuevos sectores de actividad económica claves para estudiar el proceso innovador y la actividad económica de la región; y c) ampliar el número de firmas a partir de la encuestación individualizada de los establecimientos.

- Incorporación del establecimiento como unidad de análisis en aquellas firmas cuya sede social se encuentre fuera de nuestra región.

- Temporalidad bianual de la estadística, al igual que en la Propuesta 1.

- Coste medio de la estadística ya que el INE asumiría la encuestación de aquellas empresas andaluzas incluidas en su muestra.

- Desde el punto de vista espacial, si dispusiéramos de los datos desagregados a nivel de empresa o establecimientos, sea éste industrial o de servicios avanzados, podríamos estudiar los impactos que tienen lugar en el espacio en la mayoría de núcleos/zonas donde se localicen firmas/establecimientos innovadores; las interrelaciones territoriales de cada una de las variables propuestas por el INE y parcialmente los flujos resultantes. 


\section{INCONVENIENTES}

- Necesaria coordinación con el INE para que facilitara los datos correspondientes a Andalucía.

- Continuas modificaciones en la metodología del INE, al igual que en la Propuesta 1.

- Dificultades a efectos de comparabilidad de la estadística regional con la nacional e internacional en el caso de la propuesta que contempla la incorporación nuevas empresas y/o sectores no estudiados por el INE (por ejemplo, sector servicios avanzados).

- Posibles problemas en el desarrollo de las tareas de recogida de información, por cuestiones de duplicidad. La recogida de información directa a los establecimientos, puede producir una reiteración en el desarrollo de esta tarea por parte de los organismos estadísticos implicados, en tanto éstos no puedan suministrar autónomamente esa información y remitan los cuestionarios a sus respectivas sedes sociales.

\subsection{Propuesta 3. Coordinación con el Instituto de Fomento de Andalucía (IFA) para la realización y explotación del Inventario de Recursos Tecnológicos de Andalucía (IRTA)}

En este escenario se trata de plantear una posible colaboración con el IFA para la utilización y enriquecimiento de la base de datos del IRTA, así como su posible explotación estadística. Este planteamiento surge de las posibilidades de utilización de una infraestructura consolidada por parte del IFA (recursos humanos y financieros, conocimiento de la realidad empresarial andaluza,...) y de la oportunidad que se nos presenta de efectuar esta aproximación a la realidad del proceso innovador andaluz sin la necesidad de emplear costes elevados.

El IRTA es un inventario que se elaboró por primera vez en 1992 como respuesta del interés de los principales actores implicados (IFA, Instituto Andaluz de Tecnología y Centro de Enlace sur de Europa-Andalucía -CESEAND-) por conocer el proceso de innovación tecnológica e industrial en la Comunidad Autónoma Andaluza. En 1996 se retomó este Inventario, actualizándose. Los criterios para la incorporación de empresas y entidades innovadoras en este inventario han sido: la obtención de subvenciones con cargo al V Programa Marco de la UE (1998-2002), los Programas subuencionados por el CDTI, los Programas del IFA, los Programas de colaboración con las universidades integradas en el Plan Andaluz de Investigación y otros programas propios. Cabe puntualizar que el IRTA es, en sentido estricto, un inventario y como tal no posee un carácter estadístico. 
Este inventario contiene un total de 1.297 registros y se estructura en 7 grupos de entidades: Empresas innovadoras, Grupos de investigación de las universidades andaluzas, Centros públicos de investigación, Centros tecnológicos, Instituciones públicas y Otras instituciones. Los campos contenidos en la base de datos suministrada son los siguientes: Entidad, Dirección, Código Postal, Ciudad, Provincia, Tipo de Entidad (Tamaño según número de empleados) y código CNAE. Del total de registros se deben excluir los correspondientes a Centros de Investigación, Centros Tecnológicos y Entidades Colaboradoras ya que ninguna de estas entidades se ajusta al concepto de empresa.

El IFA realiza un pequeño cuestionario para incluir nuevas firmas a su directorio. Recaba, principalmente, datos identificativos aunque también alguna información tecnológica (principales recursos tecnológicos de que dispone la empresa, tecnología que desarrolla la empresa, líneas de investigación/innovación que posee y si la empresa es titular de patentes y/o modelos de utilidad, especificando en caso afirmativo el número y referencia concreta). De esta forma, si asumimos el cuestionario del IFA como modelo de encuesta para la estadística sobre innovación tecnológica en Andalucía sería necesario enriquecerlo incorporando variables como

- Tipo de innovaciones tecnológicas desarrolladas por la empresa: producto y/o proceso.

- Personal empleado en actividades de I+D

- Carácter sistemático o no sistemático de la I+D.

- Gastos en innovación, discriminando según distintas actividades innovadoras.

- Introducción de innovaciones no tecnológicas.

- Participación en Programas de I+D e Innovación.

- Redes de innovación de la que forma parte la empresa

- Socios que constituyen las redes

VENTAJAS

- Elaboración de una estadística propia de la C.A. de Andalucía que integraría, tanto a empresas andaluzas y establecimientos, como a firmas de todos los sectores de actividad, inexistente hasta el momento.

- Facilidad para la actualización continua de información y, por tanto, garantía para la periodicidad de la estadística ya que el IFA cuenta con recursos humanos destinados, exclusivamente, a esta tarea. 
- Bajo coste en la elaboración de esa estadística debido a que se parte de un directorio existente (IRTA) que se actualiza continuamente.

- Posibilidad de estudio del subsistema científico por tipo de entidades, sectores y localización.

Tabla 3. Clasificación CNAE de las actividades empresariales contemplada por el IRTA

\begin{tabular}{|c|c|c|}
\hline SECTORES CNAE* & ACTIVIDADES ECONÓMICAS & EMPRESAS (\%) \\
\hline A & AGRICULTURA, GANADERIA, CAZA Y SELVICULTURA & $5,77 \%$ \\
\hline $\mathrm{B}$ & PESCA & $1,76 \%$ \\
\hline CA & Extracción de productos energéticos & $0,59 \%$ \\
\hline $\mathrm{CB}$ & Extracción de otros minerales, excepto productos energéticos & $1,27 \%$ \\
\hline DA & Industria de la alimentación, bebidas y tabaco & $11,35 \%$ \\
\hline DB & Industria textil y de la confección & $2,05 \%$ \\
\hline DC & Industria del cuero y del calzado & $0,49 \%$ \\
\hline DD & Industria de la madera y del corcho & $0,49 \%$ \\
\hline $\mathrm{DE}$ & $\begin{array}{l}\text { Industria del papel; edición, artes gráficas y reproducción de } \\
\text { soportes grabados }\end{array}$ & $1,76 \%$ \\
\hline DF & Refino de petróleo y tratamiento de combustibles nucleares & $0,29 \%$ \\
\hline DG & Industria química & $5,48 \%$ \\
\hline $\mathrm{DH}$ & Industria de la transformación del caucho y materias plásticas & $2,54 \%$ \\
\hline DI & Industrias de otros productos minerales no metálicos & $4,11 \%$ \\
\hline DJ & Metalurgia y fabricación de productos metálicos & $5,68 \%$ \\
\hline DK & Industria de la construcción de maquinaria y equipo mecánico & $5,19 \%$ \\
\hline DL & Industria de material y equipo eléctrico, electrónico y óptico & $3,82 \%$ \\
\hline DM & Fabricación de material de transporte & $2,45 \%$ \\
\hline DN & Industrias manufactureras diversas & $1,37 \%$ \\
\hline E & PRODUCCIÓN Y DISTRLBUCIÓN DE ENERGIA ELÉCTRICA, GAS Y AGUA & $2,74 \%$ \\
\hline $\mathrm{F}$ & CONSTRUCCIÓN & $3,52 \%$ \\
\hline G & $\begin{array}{l}\text { COMERCIO; REPARACIÓN DE VEHÍCULOS DE MOTOR, } \\
\text { MOTOCICLETAS Y CICLOMOTORES, Y ARTÍCULOS PERSONALES } \\
\text { Y DE USO DOMÉSTICO }\end{array}$ & $6,16 \%$ \\
\hline $\mathrm{H}$ & HOSTELERÍA & $0,10 \%$ \\
\hline I & TRANSPORTE & $2,84 \%$ \\
\hline $\mathrm{J}$ & INTERMEDIACIÓN FINANCIERA & $0,29 \%$ \\
\hline $\mathrm{K}$ & $\begin{array}{l}\text { ACTIVIDADES INMOBILIARIAS Y DE ALQUILER; SERVICIOS } \\
\text { EMPRESARIALES }\end{array}$ & $23,87 \%$ \\
\hline $\mathrm{M}$ & EDUCACIÓN & $0,20 \%$ \\
\hline $\mathrm{N}$ & ACTIVIDADES SANITARIAS Y VETERINARIAS, SERVICIOS SOCLALES & $0,39 \%$ \\
\hline \multirow[t]{3}{*}{$\mathrm{O}$} & OTRAS ACTIVIDADES SOCTALES Y DE SERVICIOS PRESTADOS & \\
\hline & A LA COMUNIDAD; SERVICIOS PERSONALES & $0,98 \%$ \\
\hline & Otras & $2,45 \%$ \\
\hline Total general & & $100,00 \%$ \\
\hline
\end{tabular}

Fuente: Elaboración propia a partir del IFA 


\section{INCONVENIENTES}

- Pobreza de información relativa al proceso de innovación de Andalucía, sobre todo en el supuesto de asumir el cuestionario tipo diseñado por el CESEAND debido al escaso número de variables cuantificables por lo que sería necesario su enriquecimiento.

- Los resultados no son comparables con la estadística nacional y/o internacional.

- El IRTA contiene registros referidos a entidades que no son empresas y deben ser excluidos.

- Aunque el IRTA contiene un elevado número de empresas innovadoras andaluzas, superior a la muestra del INE para nuestra región, no recoge la totalidad de éstas, por lo que es necesario crear un directorio donde se incluya la población total de firmas innovadoras andaluzas.

- A pesar de que este directorio se actualiza de forma continua, bianualmente el IFA recoge un cuestionario de los registros del IRTA que tan sólo es respondido por el $10 \%$ de éstas, lo cual da idea de la cautela con que habría que utilizar esta fuente.

- Al disponer únicamente de datos de carácter nominal del subsistema científico, tecnológico y productivo innovador no cabe hacer un estudio de los flujos del sistema ciencia tecnología industria. Por otra parte, el análisis espacial de cada uno de los subsistemas sería de corto alcance, dado que el número de variables incorporadas es muy reducido.

\subsection{Propuesta 4. Elaboración de una estadística propia, de dimensión regional, sobre innovación tecnológica a partir del método descendente}

Se trata de proponer una metodología para la generación de una estadística sobre innovación empresarial en Andalucía, cuyo principal objetivo es obtener información directa sobre el proceso de innovación tecnológica en las empresas andaluzas, elaborando indicadores que permitan conocer los distintos aspectos del proceso innovador (impacto económico territorial y sectorial, características espaciales y no espaciales de las actividades innovadoras, coste, etc.). Esto implica, necesariamente, el desarrollo de un directorio de empresas innovadoras andaluzas como base para la construcción de dichas estadísticas.

Esta propuesta metodológica consiste en la elaboración de una estadística sobre innovación empresarial para Andalucía que asuma parcialmente la metodología del 
INE teniendo en cuenta que el ámbito de estudio se extendería a todas las empresas y establecimientos que radican en Andalucía con al menos una persona ocupada remunerada, cuya principal actividad económica se corresponde con cualquiera de los sectores CNAE, incluidos el agrario y los de servicios.

\section{VENTAJAS}

- Adecuación de la población a la realidad innovadora andaluza ya que se parte de un directorio nuevo que incorporaría la mayoría de las empresas y/o establecimientos innovadores andaluces.

- Incorporación del establecimiento como unidad de análisis que posibilita un mejor conocimiento de la estructura del proceso innovador en Andalucía.

- La distribución territorial de las empresas innovadoras se ajusta a la realidad económica andaluza.

- Temporalidad bianual de la estadística coincidiendo con la del INE.

- Desde un punto de vista espacial se puede hacer un análisis territorial, y de forma parcial un estudio de interrelaciones espaciales y de flujos, al disponer de datos precisos sobre la empresa que constituye el origen del flujo.

\section{INCONVENIENTES}

- Dificultades a efectos de comparabilidad de la estadística regional con la nacional e internacional aunque se puede evitar tabulando aparte los sectores no contemplados por el INE.

- Posibles dificultades en el desarrollo de las tareas de recogida de información, por duplicidades ya que una misma empresa puede ser encuestada por diferentes organismos.

- Coste alto de la estadística debido a que el organismo encargado de ésta deberá asumir todos los costes, humanos y económicos, para llevarla a cabo.

- Información incompleta y de carácter discreto y ordinal sobre los organismos o firmas constitutivos del otro extremo del flujo, mientras que se tiene un conocimiento preciso de la empresa que genera el origen de la relación. Por consiguiente no podemos medir la magnitud de los flujos que organiza el sistema tecnológico-productivo o que se originan dentro de cada uno de estos subsistemas. 


\section{Notas}

1 La OCDE presentó en la reunión del Grupo de Expertos Nacionales en Indicadores de Ciencia y Tecnología celebrada en París el día 10 de diciembre de 1990 una versión preliminar de un método para la recogida e interpretación de datos sobre innovación tecnológica (Manual de Innovación), que fue revisado a lo largo de 1991. En 1992 se llevó a cabo la difusión general de las "Directrices propuestas por la OCDE para la recogida e interpretación de datos sobre innovación tecnológica", más conociclas cono Manual de Oslo. En 1997 ha aparecido la segunda edición.

2 Generalmente los técnicos de los Institutos Estadísticos Regionales se ocupan de las estadísticas de distintos temas, pudiéndoles calificar en ese sentido de polívalentes. No se especjalizan en una o en dos materias, para ello estos Organismos deberían disponer de muchos mas recursos.

3 Los datos de la Encuesta sobre Innovación Tecnológica del INE de 1998 son los últimos publicados.

4 Debemos puntualizar que el INE habitualmente no facilita información desagregada a nivel de empresa, correspondientes a cada una de las CC. AA.

\section{Bibliografía}

Archibugi, D. (1997): Innovazione e globalizaciones. Definizione, misorazione $e$ implicazioni per la politiche, Consiglio Nazionale delle Ricerche, Roma.

Blasco Vizcaino, C., González Fajardo, F. y Podadera Rivera, P. (1998). "La innovación en el sistema productivo andaluz". II Congreso de Ciencia Regional de Andalucia. Jaén.

Camagni, R. edit. (1991): Innovation Network. Spatial perspectives. London, Belhaven Press.

Cano García, G. (1985): Aproximaciones al análisis geográfico Regional. Servicio de Publicaciones de la Lniversidad de Sevilla, Sevilla. 136 p.

Castells, M. y Hall, P. (Directores) (1992): Andalucía: innovación tecnológica $y$ desarrollo económico. Espasa Calpe. Tomos I y II. Madrid. 919 pp.

Comisión Europea (1996). La Dimensión Regional de las Estadisticas de $I+D$ e Innovación. Manual Regional. Luxemburgo. OCDE.

Dosi, G. (1992): "Fuentes, métodos y efectos microeconómicos de la innovación", Ekonomiaz, n'22, pp 269-331
Durán, A. (Coord.) (1999): Geografia de la innovación. Ciencia, tecnologia y territorio. Los libros de la catarata. Madrid. $510 \mathrm{p}$.

Felman, M. P. (1994): The Geography of Innovation. Kluwer Academic Publishers, Boston

Instituto de Estadistica de Andalucía (2001): Encuesta sobre Innovación Tecnológica en las Empresas. Resultados de Andalucia. Año 1998, http://www.iea.junta-ándalucia.es.

INE (1998). Estadistica sobre las actividades en investigación científica y desarrollo tecnológico $(I+D)$. Indicadores básicos 1996. INE, Madrid.

INE (2000). Estadistica sobre las actividades en investigación cientifica y desarrollo tecnológico $(1+D)$ 1997. INE, Madrid.

Jordá Borrell, R. (1992): Las relaciones ciencia-tecnologia-industria en Andalucia y el papel de la administración. Instituto de Desarrollo Regional. Universidad de Sevilla. Sevilla. 262 pp.

Jordá Borrell, R. (Dir.) (2000). El entorno productivo y su incidencia en el desarrollo del 
proceso innovador de las empresa andaluzas, Ed. IEA. Sevilla.

Jordá Borrell, R.; Posada Simeón, C. y Lucendo Monedero, A.L. (2000). "Resultados de la explotación de los de la Estadística de Innovación Tecnológica del INE para Andalucía". Regional Studies (en prensa).

Klíne, L. y Rosenberg, N. (1986). "An overview of innovation" en R. Landau y $N$. Rosenberg (Eds) The Positive Sum Strategy. Harnessing Technology for Economic Growth (Washington DC. National Academy Press), pg. 135-148.

Lundvall, B. A. (1992): National systems of innovation: towards a theory of innovation and interactive learning, Pinter, London.

Morrill, R. H., Gaile, G. y Thrall, G. I. (1988): Spatial diffussion. Sage Publications, London.

Nelson, R. y Winter, R. (1982): An evolutionary theory of economic change. Cambridge, Hardvard University Press. 385 p.
OCDE (1992). Proposed Guidelines for Collecting and Interpreting Technological Innovation Data. Oslo-Manual. París.

OCDE (1994): Proposed Standard Practice for Sumeys of Research and Experimental Development. Frascati-Manual. París.

Patel, P. y Pavitt, Q. (1995): "Patterns of technological activity: their measurement and interpretation" Stoneman (ed): Handbook of the economics of innovative and technological change, Oxford, Blackwell

Pomares Hernández, I. (1998): Comportamientos innovadores de las empresas industriales en Andalucia. Madrid, Civitas.

Santos, M. (2000): La naturaleza del espacio. Ed. Ariel Geografía, Barcelona

Storper, (1995): "Regional technology coalitions: an essential dimension of national technology policy", Research Policy, n 24 , pg. 895-911.

Veltz, P. (2000): Mundialización, ciudades y Territorio. Ed. Ariel Geografia, Barcelona. 